\title{
Protección Multi-robot de Infraestructuras: Un Enfoque Cooperativo para Entornos con Información Limitada
}

\author{
David Garzón-Ramos ${ }^{1,2}$, Mario Garzón Oviedo ${ }^{1}$ y Antonio Barrientos ${ }^{1}$ \\ ${ }^{1}$ Centro De Automática y Robótica UPM-CSIC \\ Calle José Gutiérrez Abascal, 2. 28006 Madrid, España \\ ${ }^{2}$ GTA Propiedades Ópticas de los Materiales, Universidad Nacional de Colombia - Sede Manizales \\ dgarzon@etsii.upm.es, ma.garzon@upm.es, antonio.barrientos@upm.es
}

\section{Resumen}

Se presenta el diseño, implementación y evaluación de un sistema multi-robot que aporta al aseguramiento de zonas con acceso restringido en infraestructuras, a través de la interceptación de intrusos. Éste, se basa en el aprovechamiento del algoritmo de Fast Marching y provee una solución cooperativa multi-robot capaz de funcionar en condiciones de información limitada. El sistema ha sido probado en escenarios sintéticos y reales, alcanzando en todos los casos la interceptación del objetivo mediante una respuesta cooperativa.

Palabras clave: Distribución Dinámica de Área, Fast Marching, Predicción de Trayectorias, Sistemas Multi-Robot.

\section{INTRODUCCIÓN}

Continuamente se han buscado medios que permitan mejorar las capacidades de operación de robots móviles en tareas relacionadas a la protección de infraestructuras, destinando múltiples estudios al problema de protección cooperativa a través de robots trabajando simultáneamente para atrapar a uno o varios evasores en situaciones de riesgo [1]. En varios casos se emplean técnicas que buscan soluciones complejas y requieren un máximo de información, parametrización y aprendizaje mediante la implementación de algoritmos genéticos [2], redes neuronales [3] y la optimización de la asignación de tareas dentro del MRS[4] para generar alianzas entre los agentes del sistema multi-robot y obtener comportamientos más eficientes dentro de la captura de objetivos.

Es así como, los sistemas multi-robot (MRS) y las estrategias de cooperación surgen como respuesta a dicha problemática, debido a la capacidad que brindan para responder de forma simultánea y coordinada, ante vulneraciones de seguridad como la presencia de intrusos en lugares de acceso restringido. Por esta razón, el objetivo de este trabajo es entablar un mecanismo eficiente de interceptación cooperativa, optimizando la respuesta propia de cada robot ante las amenazas mediante la distribución dinámica de áreas de acción y la predicción de trayectorias del mismo.
Nuestro trabajo difiere en que no solo propone estrategias de búsqueda y captura de objetivos, plantea una arquitectura multi-robot funcional, también estima la probabilidad de trayectoria de los intrusos y genera una estrategia coordinada de respuesta ante las amenazas en condiciones de información limitada.

Las siguientes secciones del documento se estructuran así: En la sección 2 se describe la metodología empleada en el MRS. En la sección 3 se definen los experimentos desarrollados para validar la propuesta. La sección 4 se hace un análisis de los resultados obtenidos y finalmente en la sección 5 se muestran las conclusiones del trabajo así como líneas futuras de desarrollo.

\section{METODOLOGÍA}

La base del MRS presentado en este trabajo se encuentra en el aprovechamiento del Método de Fast Marching (FMM)[5] en la ejecución de dos tareas independientes: realizar la distribución dinámica del mapa como espacios de trabajo para cada robot y obtener una estimación de la probabilidad de que los intrusos sigan cualquier trayectoria dentro del mapa. Por este motivo, se ha desarrollado la arquitectura de software denominada Fast Marching Method Core (FMM Core), esta concentra el procesamiento de información en el algoritmo FMM, así se obtienen bajos tiempos de respuesta y se evita el intercambio de información entre diferentes instancias. En la Figura 1 se puede ver el esquema del FMM Core.

Una de las mayores ventajas del sistema propuesto es su mínimo requerimiento de información, por esto el MRS solo tiene como entradas un mapa bidimensional del lugar en donde se desarrolla la tarea, las posiciones de los robots y de los intrusos cuando estos son detectados (en adelante se denominará a esta posición como objetivo). A partir de esto es capaz de generar trayectorias independientes en cada robot, y que estas respondan a una acción cooperativa.

Primero se obtiene un mapa de costos representativo del entorno, en esta etapa se disminuye la resolución del mapa para agilizar el procesamiento de información y luego, este es transfor- 


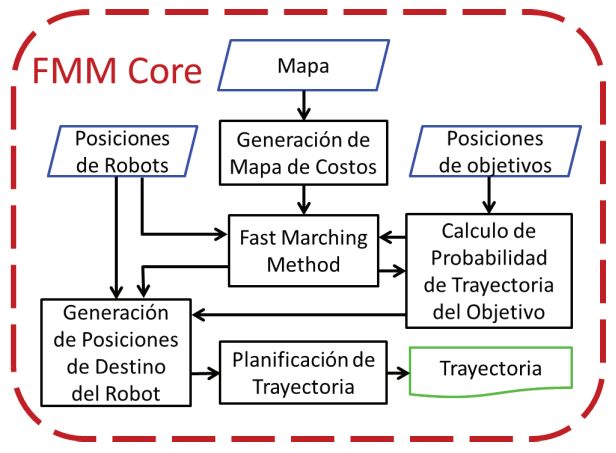

Figura 1: FMM Core - Arquitectura de software.

mado añadiendo costos crecientes a lugares cercanos a paredes u obstáculos. De este modo, en todos los procesos asociados al FMM Core se toma en cuenta la planificación de rutas con evasión de obstáculos de los robots como de los intrusos.

En las siguientes subsecciones se da una idea general sobre cómo el FMM Core ejecuta las tareas de distribución dinámica de áreas y predicción, y cómo estas en conjunto, permiten llevar a cabo labores de interceptación cooperativa multi-robot.

\subsection{ARQUITECTURA MULTI-ROBOT}

El MRS fue desarrollado mediante el esquema de trabajo de Robot Operating System (ROS), de esta forma se aprovecharon sus de herramientas y paquetes de software para lograr la completa puesta en marcha del sistema. En primera instancia, ROS permite una fácil implementación de una arquitectura multi-robot a través de los nodos de master, éste es capaz de almacenar y manejar toda la información que debe transferirse dentro de cada robot y entre varios robots también. Sin embargo, esta herramienta se queda corta en casos donde los sistemas crecen en complejidad y/o la cobertura completa de las comunicaciones no está asegurada.

En este trabajo se ha empleado una arquitectura multi-master, en donde cada participante del MRS conserva las capacidades brindadas por los nodos master y a su vez, se evitan las debilidades presentadas por la centralización de los recursos. El esquema de la arquitectura Multi-master empleada se muestra en la Figura 2.

Dentro del MRS se pueden identificar tres tipos de agentes: Robots, Objetivos y la Estación Base. Los robots se encargan de realizar la tarea de interceptación del intruso cuando éste es detectado. Cada uno cuenta con un sistema propio de localización que recoge la información aportada por su odometría, unidades medida inercial (IMU) y el sistema de posicionamiento global (GPS) y la fusiona mediante un Filtro del

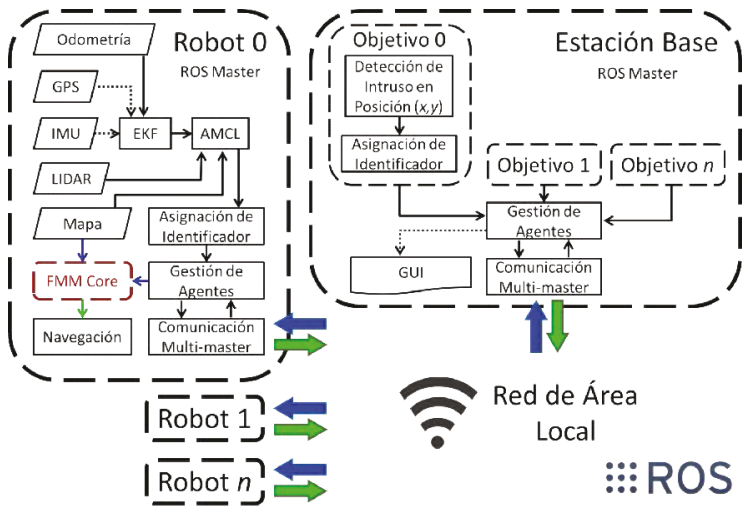

Figura 2: Arquitectura multi-robot

Kalman Extendido (EKF). Dado que el MRS está pensado para funcionar tanto en grandes infraestructuras que suelen contar con espacios abiertos, lugares sin cobertura GPS, ambientes con alteraciones magnéticas, entre otros, el uso de la IMU y el GPS son opcionales; tomando en consideración que errores en el posicionamiento decaen en errores en la interceptación cooperativa. Para solventar este problema, adicionalmente se han incluido las medidas aportadas por el LIDAR en el paquete AMCL (Adaptative Monte Carlo Localization) de ROS, el cual toma el mapa original y mejora la localización del robot en función del reconocimiento de su entorno.

La posición estimada por cada robot se marca con un identificador propio y es comunicada en forma de broadcast mediante el gestor de agentes. Éste, además de transmitir la posición propia, escucha el broadcast y recoge la posición de otros robots y de los objetivos. A continuación, relaciona la posición con su identificador y entrega una lista de posiciones de robots y de objetivos al FMM Core. Una vez procesada la información, el FMM Core entrega una trayectoria para que el sistema de navegación controle el desplazamiento del robot. El gestor de agentes es el único componente que transfiere información al paquete de multi-Master, de este modo se mantiene al mínimo el intercambio de datos entre agentes y se optimiza desempeño de las comunicaciones.

El segundo tipo de agente son los objetivos. Como se mencionó anteriormente, un objetivo se define como una posición en donde se ha detectado un intruso. En la práctica estos agentes están asociados a sistemas de detección a través de movimiento, temperatura, entre otros. Cuando un intruso es detectado, el MRS marca la posición del objetivo con un identificador y transmite la información hacia el gestor de agentes. En este trabajo se ha planteado que los sistemas de detección son simples, constituidos por redes de sensores que solo tienen la capacidad de detectar 
la presencia de un intruso en su área. Por este motivo solo se requiere un gestor de agentes ubicado en la estación base para manejar la información proveniente de todos los objetivos.

El último tipo de agente es la estación base. Este es el punto en donde se concentra la información de los objetivos y es transmitida hacia los robots. Cuenta con su propio nodo master y permite visualizar la información proveniente del MRS a través de la RVIZ, la interfaz gráfica de usuario (GUI) propia de ROS. Al igual que los robots, únicamente transmite la información proveniente de su gestor de agentes a las comunicaciones multi-master.

\subsection{DISTRIBUCIÓN DINÁMICA DE ÁREA}

El algoritmo de FMM permite determinar el tiempo mínimo de llegada de una onda, que se desplaza con una velocidad determinada, desde un punto de origen a cualquier otro punto en el espacio. Cuando la velocidad es constante, entonces es posible relacionar directamente el tiempo de llegada con la distancia recorrida. De forma análoga, el algoritmo puede ser empleado para determinar la distancia mínima que un robot holonómico con velocidad constante debe recorrer para llegar a cualquier otro punto.

En el caso de la onda, si en lugar de tener un solo punto de origen se tienen dos, las ondas se expandirán hasta encontrar una frontera en donde los frentes de onda se encuentran. Esta frontera describe la distancia máxima a la que la una onda puede llegar antes que su semejante. Si mantenemos la analogía y en vez de tener un solo robot, planteamos el caso con varios de ellos, la frontera generada por el FMM describe el área máxima a la que un robot puede llegar antes que cualquier otro robot.

Este principio se emplea dentro del FMM Core para realizar la distribución dinámica de área. El FMM Core recibe la posición de todos los robots y ejecuta el algoritmo FMM con dichas posiciones como orígenes. Así, puede realizar una segmentación y asignación de áreas correspondiente a la máxima distancia de recorrido a la que un robot puede llegar antes que cualquier otro dentro del MRS. A medida que los robots se desplazan, la distancia máxima va cambiando en función a su cercanía con otros robots, y en consecuencia, el área asignada también va cambiando. El procesamiento y distribución dinámica se realiza mediante el procesamiento del mapa de costos como imagen, por tanto este proceso es completamente escalable y no se altera el costo computacional dado que la resolución del mapa de costos que ingresa
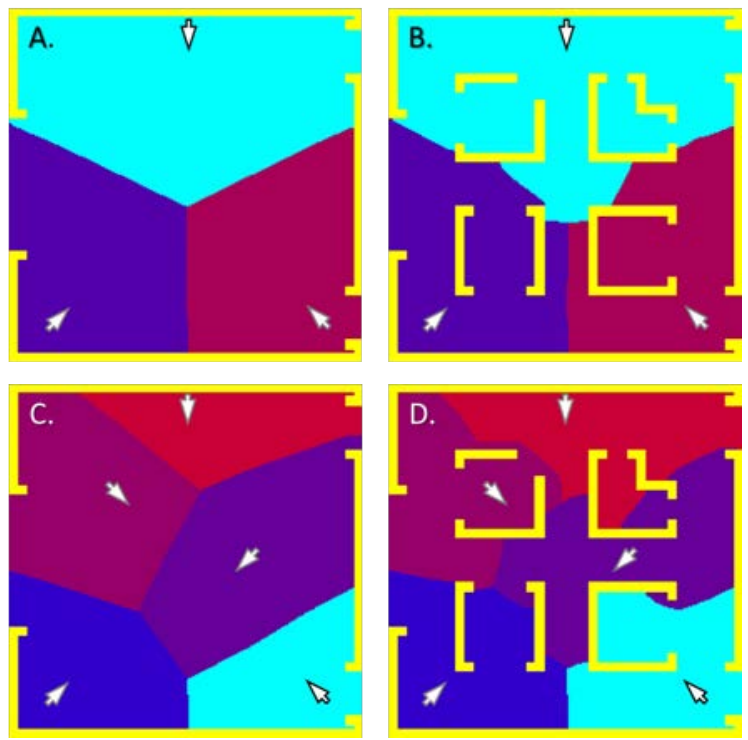

Figura 3: Distribución dinámica de área: A. Con tres robots. B. Con tres robots y obstáculos. C. Con cinco robots. D. Con cinco robots y obstáculos.

al FMM se mantiene igual.

En la Figura 3 se puede observar la distribución dinámica de área. Las imágenes corresponden a mapas artificiales en donde las zonas amarillas representan paredes u obstáculos, las flechas representan a los robots y cada área coloreada corresponde a la distancia máxima a la que cada robot puede llegar antes que cualquiera de los demás. Dicha segmentación fue realizada mediante el FMM Core. En las figuras 3.A. y 3.C. se puede apreciar como la distribución cambia al aumentar el número de robots. En las figuras 3. B. y 3.C. se observa que para una misma configuración de robots, la inclusión de obstáculos y paredes cambian su área de acción debido a que estos limitan su desplazamiento.

\subsection{PREDICCIÓN DE TRAYECTORIAS}

La predicción de trayectorias se explora con mayor profundidad en [6], pero en esta sección se da una visión general para efectos de completitud. El FMM Core también se encarga de realizar una estimación de la probabilidad que un intruso detectado se desplace por cualquier trayectoria en el entorno. Esta predicción se realiza empleando únicamente la posición del sensor que ha detectado al intruso y la información aportada por el mapa, por tanto se asume que puede desplazarse en cualquier dirección y que lo realiza a una velocidad constante. A través del algoritmo de FMM se determinan los posibles tiempos de llegada del intruso desde la posición de origen hasta cualquier 
otro punto. Posteriormente, a partir de una discretización arbitraria de los tiempos de llegada se hace una representación del mapa por grafos, agrupando en cada nodo las áreas que estén conectadas espacialmente y que también se encuentren dentro de un mismo lapso de tiempo. De este modo, se obtiene un grafo dirigido que inicia en el punto de detección del intruso y se extiende por todas las posibles trayectorias que éste puede recorrer. A continuación, se emplea dicho grafo para desplegar una distribución de la probabilidad sobre todo el mapa. Así, el primer nodo recibe un $100 \%$ de probabilidad de encontrarse en la trayectoria del intruso, y de ahí, en cada nodo se realiza una división equitativa de la probabilidad según sea el número de nodos hijos asociados en el grafo. En los casos en que una zona puede ser alcanzada por diferentes trayectorias, es decir, que tiene varios nodos padres, su probabilidad esta descrita por:

$$
P(a \cup b)=P(a)+P(b)-P(a \cap b)
$$

dado que $a$ y $b$ son eventos independientes y mutuamente excluyentes entonces la probabilidad del nodo es

$$
P(a \cup b)=P(a)+P(b)
$$

Esta expresión representa la suma de las probabilidades de los nodos padres en el nodo hijo, la cual se asocia a cada pixel del mapa representado por dicho nodo. Un proceso similar se sigue en los casos en que hay varios intrusos, y por tanto se fijan varios objetivos para el MRS. Primero se calcula el mapa de probabilidades para cada objetivo por separado, y a continuación, se realiza la suma de probabilidades en cada pixel mediante la expresión (1). Dado que los eventos son independientes pero no mutuamente excluyentes, la probabilidad total en cada pixel está determinada por:

$$
P(a \cup b)=P(a)+(b)-P(a) *(b)
$$

En la Figura 4 se puede observar la distribución de probabilidades cuando uno o varios objetivos son detectados. Las imágenes corresponden a mapas sintéticos en donde el origen se indica con una alerta roja, las zonas claras representan alta probabilidad, las zonas oscuras se asocian a una baja probabilidad y las zonas negras son obstáculos y paredes. Las figuras 4.A., 4.C. y 4.E. muestran la distribución de probabilidades para un mapa en donde aparecen dos diferentes intrusos y luego se realiza la suma de sus probabilidades. Las figuras 4.B., 4.D. y 4.F. permiten observar como la inclusión de obstáculos genera un mayor numero de rutas posibles, lo que se traduce en una división más compleja de las probabilidades. En a figura 4.A. la probabilidad de $100 \%$ se divide
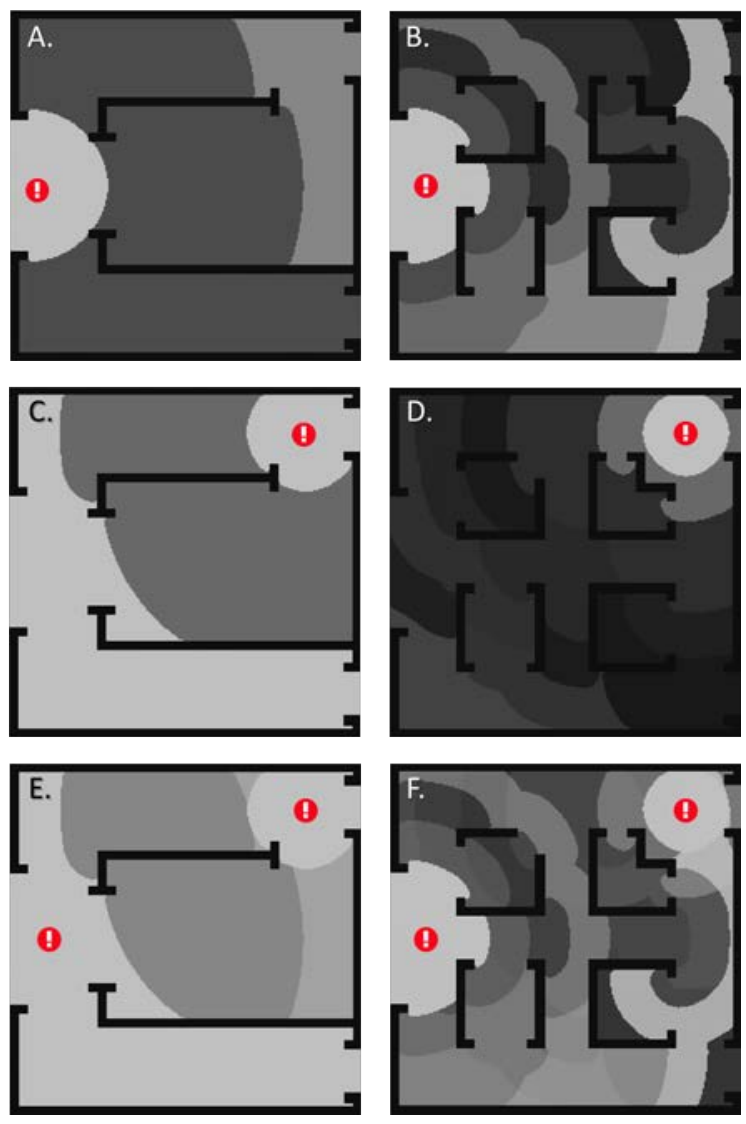

Figura 4: Predicción de trayectorias: A. Con objetivo 1. B. Con un objetivo 1 y obstáculos. C. Con objetivo 2. D. Con objetivo 2 y obstáculos.C. Con objetivos 1 y 2 . D. Con objetivos 1 y 2 y obstáculos.

en tres debido a las tres posibles rutas que puede seguir el intruso. Posteriormente la ruta superior y central se unen y por tanto sus probabilidades se suman. En la figura 4.C. el intruso solo tiene dos opciones de trayectoria, por esto la probabilidad no decrece tanto como en la figura 4.A. de igual forma, cuando las rutas se encuentran nuevamente, la probabilidad crece. Finalmente, en la figura 4.E. se muestra la suma de probabilidades obtenida de los dos objetivos. Se puede apreciar como la probabilidad se incrementa para todo el mapa, lo que es correspondiente al incremento al numero de individuos a capturar.

\subsection{INTERCEPTACIÓN COOPERATIVA}

A partir del mapa original y de los procesos de distribución de área y de predicción de trayectorias, el FMM Core genera cuatro nuevos mapas diferentes: un mapa de distancias de cada punto al robot más cercano (MDR) obtenido al ejecutar el FMM con las posiciones de los robots como orígenes; un mapa de segmentación por áreas 

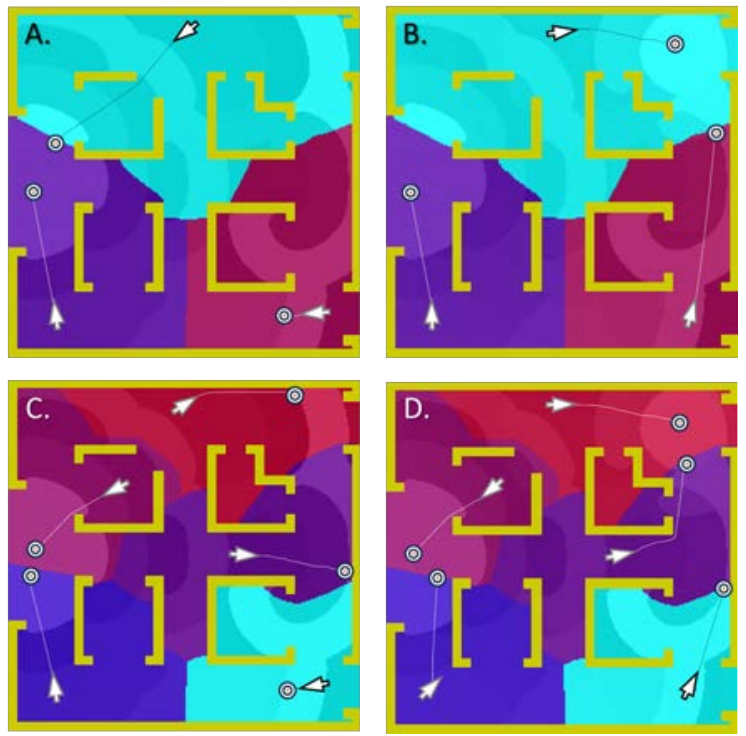

Figura 5: Interceptación cooperativa: A. Con tres robots y un objetivo. B. Con tres robots y dos objetivos. C. Con cinco robots y un objetivo. D. Con cinco robots y dos objetivos.

(MSA) asignadas a cada robot como resultado del proceso de distribución dinámica de área; un mapa de distancia de cada punto al objetivo más cercano (MDO) que se obtiene al cruzar los mapas obtenidos empleando el FMM con la posición de cada objetivo como origen; y finalmente, un mapa de segmentación de áreas por probabilidades (MSP) de la aplicación de la etapa de predicción de trayectorias.

La información contenida en estos cuatro mapas es empleada por cada robot para determinar el destino que optimiza la probabilidad de interceptar un objetivo dentro de su actual área de acción. Esta selección se realiza siguiendo los siguientes pasos:

1. En el MSA busca el valor con el que han sido marcados los pixeles en posiciones contiguas para determinar su identificador de área de acción.

2. En el MSP busca el mayor valor de probabilidad asociada a los pixeles que comparten su identificador de área de acción.

3. En el MDO busca el menor valor de distancia en los pixeles que tienen un mayor valor de probabilidad en el MSP dentro de su área de acción.

4. En el MSA busca el menor valor de distancia en los pixeles que tienen un menor valor de distancia en el MDO, un mayor valor de probabilidad en el MSP y que están dentro de su área de acción.
De esta forma, cada robot busca dirigirse al punto dentro de su área de acción, con mayor probabilidad de interceptación, que se encuentra más cerca de un objetivo y que finalmente se encuentra más cerca de sí mismo. La posición de destino es ingresada un planificador de tipo Dijkstra para obtener la trayectoria que debe seguir el robot. Este comportamiento se mantiene de forma dinámica, dado que el desplazamiento de los robots y de los objetivos, genera constantes cambios en las áreas de acción y las probabilidades, brindando así nuevos destinos que puedan mejorar la posición actual. Gracias a que el FMM Core se aloja en cada robot y a la continua transmisión de las posiciones de los agentes, los robots mantienen un estado sincronizado y equivalente en todo el MRS.

Dado que los robots operan en conjunto para alcanzar una tarea global, es posible clasificar al MRS como un sistema cooperativo [7], en donde existe un conocimiento constante sobre la existencia de sus coagentes, presentan un nivel fuerte de coordinación determinado por el protocolo de decisión que toma en cuenta las acciones de todo el MRS, y que finalmente se ejecuta de forma distribuida y autónoma en cada robot. La acción cooperativa se alcanza debido a que los robots no van directamente hacia el objetivo, por el contrario, buscan optimizar su posición dentro de su área. Esto se traduce en que, el MRS se distribuye para ocupar sitios estratégicos que aumenten para cada uno la probabilidad de interceptar al intruso, sin que dos robots puedan mejorar su posición mediante el mismo destino y la misma trayectoria.

En la Figura 5 se muestra la superposición de los mapas MSA y MSP de las Figuras 3 y 4, en este se han generado los destinos para cada robot y su correspondiente trayectoria en el momento inicial. Cada color dentro del mapa corresponde al área de acción asociada a un robot, en donde los tonos más claros corresponden a zonas de mayor probabilidad. Las flechas representan posición actual de los robots y los puntos señalan las posiciones de destino asociadas. En las figuras 5.A. y 5.B. se han empleado tres robots para interceptar a uno y dos objetivos respectivamente. Se puede apreciar como ante el cambio de probabilidades el MRS cambia su comportamiento con el fin de lograr la mayor eficiencia en la tarea. En las figuras 5.C. y 5.D., se aumenta a cinco el número de robots. En este caso, la distribución de estos es más estratégica y logran abarcar de mejor manera las posibles trayectorias de los objetivos.

\section{DETALLES EXPERIMENTALES}

En el experimento se emplearon dos plataformas robóticas móviles, los robots Summit XL y 


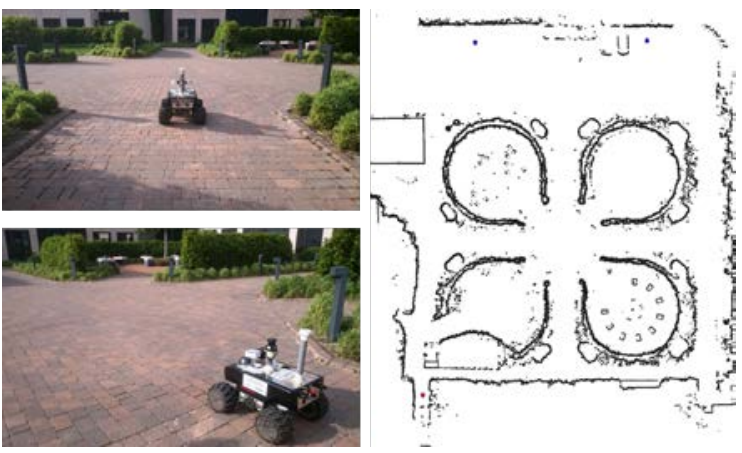

Figura 6: Escenario de pruebas: Jardines internos.

Summit HL de Robotnik®. Éstos poseen una cinemática de tipo skid-steering y están equipados con un ordenador de abordo integrado con ROS, que procesa la información recibida por los sensores y ejecuta los algoritmos de navegación y demás. También poseen un encoder en cada rueda, giróscopo de alta precisión ensamblado en el chasis, LIDAR, IMU, GPS y cámaras PTZ (Pan, Tilt and Zoom).

Para evaluar el desempeño del MRS se realizaron pruebas en dos escenarios distintos: los jardines interiores de un complejo de edificios y un aparcamiento subterráneo. Estas dos locaciones son representativas de las condiciones comúnmente encontradas en infraestructuras críticas como pueden ser acceso limitado o nulo a GPS, interferencias magnéticas y presencia de obstáculos tales como grandes columnas, coches, vegetación, entre otros.

Primero se realizó una reconstrucción bidimensional de los entornos para obtener el mapa que emplea el FMM Core, luego se ubicaron los robots en posiciones arbitrarias dentro del escenario, y finalmente desde la estación base se indicaron posiciones objetivos dentro del mapa a través de RVIZ; en consecuencia, los robots debían responder de forma cooperativa ante la presencia del intruso. En este trabajo se evaluó la aptitud del MRS para desempeñarse en entornos reales y su capacidad cooperativa en relación a trabajos previos; experimentos posteriores se encaminarán a obtener datos estadísticos sobre su eficiencia. En las Figuras 6 y 7 se muestran los entornos y el mapa durante el experimento.

\section{RESULTADOS}

En la Figura 8 se muestra un ejemplo de las trayectorias seguidas por los robots para interceptar a un intruso detectado en la parte baja del mapa de los jardines interiores. Al igual que en los mapas anteriores, el color amarillo denota zonas restringidas, los colores azul claro y purpura corresponden áreas

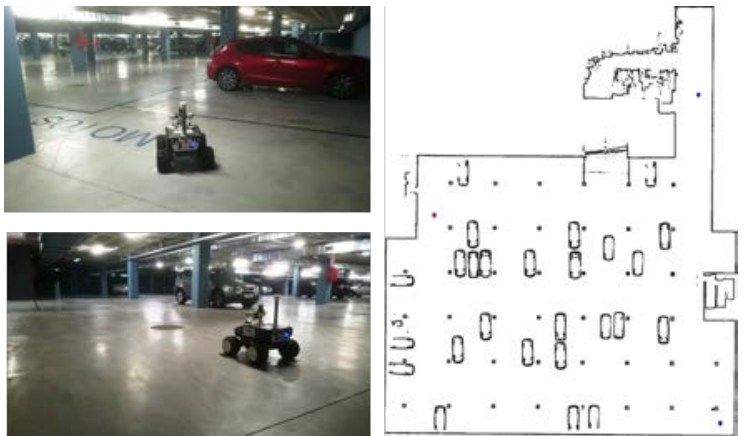

Figura 7: Escenario de pruebas: Aparcamiento subterráneo.

de acción de cada robot. Las tonalidades dentro de cada color determinan la probabilidad, en donde los tonos claros corresponden a una probabilidad mayor. La posición de los robots está indicada por las flechas, sus destinos por círculos pequeños y la posición del objetivo por una alerta roja.

En el primer instante, los robots tienen una distribución equidistante del espacio en sus áreas de acción. Éstas están representadas por los colores azul claro y purpura. Los destinos se fijan en los puntos de mayor probabilidad que se encuentran más cercanos a cada robot, esto es, llevar el robot de área purpura directamente hacia el objetivo, y al robot de área azul claro hacia el centro de los jardines. Este punto es de especial interés dada la posición actual del objetivo, debido a que desde ahí únicamente existen tres trayectorias diferentes para llegar a él; en el segundo instante el robot de área azul claro cambia su destino a uno más cercano al objetivo, esto gracias a que el desplazamiento de ambos robots le ha permitido ganar mayor área de acción y una mejor posición de interceptación; en el cuarto instante, el robot llega a su destino y aguarda ahí dado que no encuentra una mejor posición dentro de su área; en el quinto instante, el robot de área purpura disminuye su área de acción debido a que el robot de área azul es ahora quien puede llegar más rápidamente a esos lugares; y finalmente, en el sexto instante el robot de área purpura intercepta al objetivo.

En la Figura 9 está la secuencia derivada de los experimentos en el aparcamiento subterráneo. En este caso no hay una distribución evidente de la probabilidad, esto debido a que los espacios abiertos no permiten realizar una distribución tan clara de ésta; a diferencia de las bifurcaciones presentes en los caminos de los jardines en el experimento anterior. En este experimento también se aprecia como el robot de área azul claro cambia su meta en los instantes 3 y 4, debido a que la distribución dinámica de áreas le ha permitido obtener mejores posiciones de interceptación. Al igual en que en el escenario de los jardines, el robot de área azul 

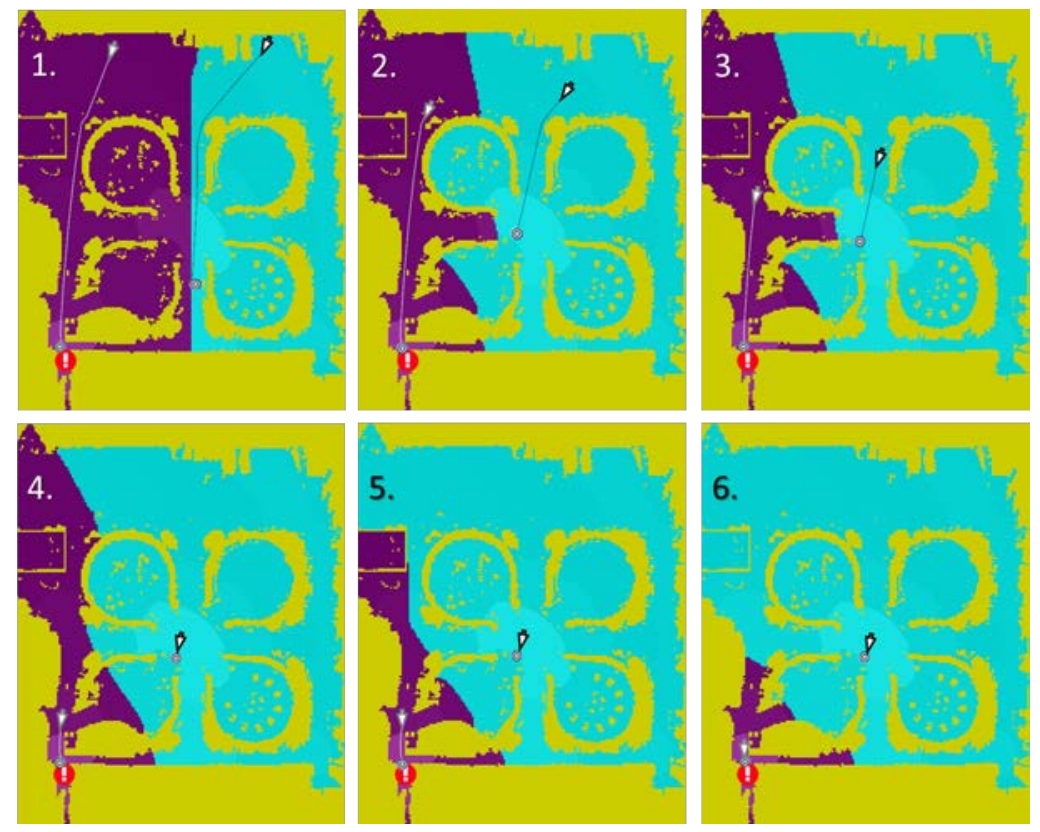

Figura 8: Secuencia del comportamiento del MRS en el escenario de los jardines.
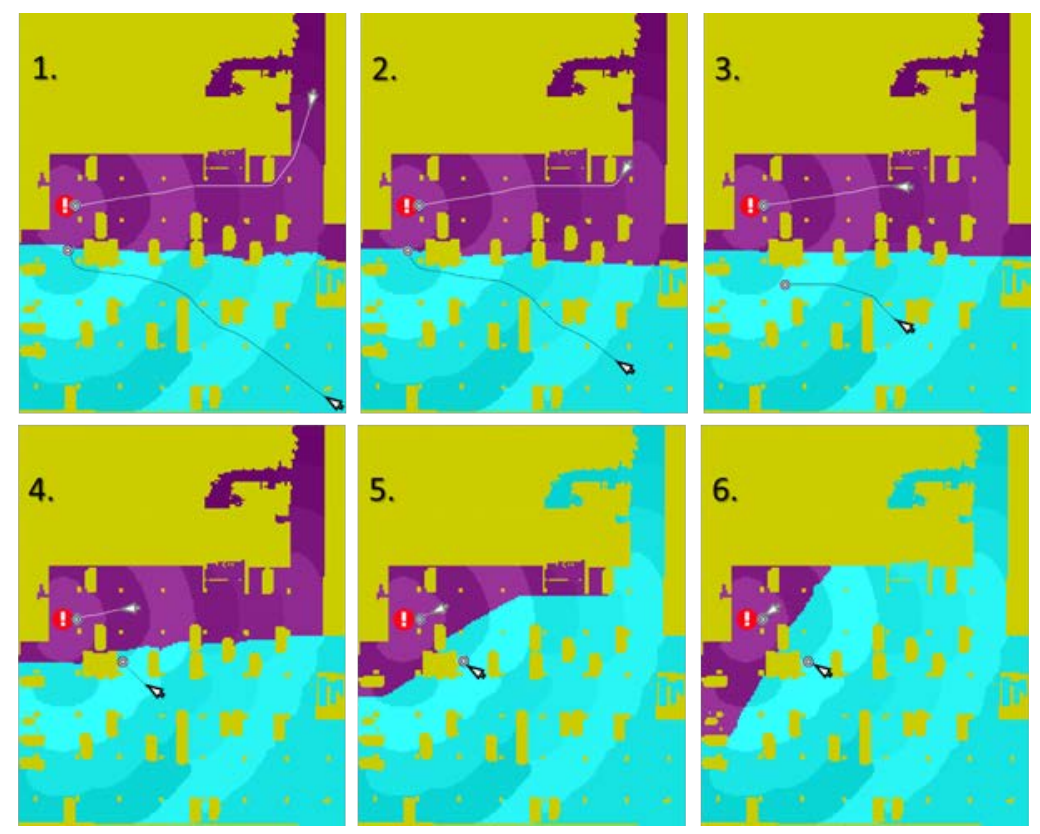

Figura 9: Secuencia del comportamiento del MRS en el escenario del aparcamiento subterráneo.

claro espera en su posición a encontrar un destino mejor que el actual.

Este es un avance significativo respecto al trabajo presentado en [8], en donde se emplean estrategias similares para realizar una repartición de áreas y la posterior interceptación del intruso. En dicho trabajo, el objetivo es incluido junto a los robots en la segmentación del área, en este caso las fronteras separan los puntos a los que el objetivo puede llegar antes que cualquier robot. Luego, los robots que comparten frontera con el objetivo se desplazan hacia ella, con el fin de minimizar las posiciones que el intruso puede ocupar sin encontrar a uno de los perseguidores. Finalmente, los robots que no comparten frontera con el objetivo se dirigen directamente hacia él a través de una ruta arbitraria trazada por el planificador.

El primer gran problema que presenta este enfoque es que la inclusión del objetivo dentro de la distribución de áreas restringe las trayectorias de los robots a trayectorias óptimas hacia los puntos que estos pueden alcanzar antes que el evasor. En cambio, en nuestra propuesta el componente cooperativo no solo asume trayectorias óptimas, 
la estrategia también permite asignar rutas no óptimas que complementen y aumenten las probabilidades de éxito de las que sí lo son. El segundo inconveniente es que los robots que no comparten frontera con el intruso siguen una trayectoria arbitraria hacia él, de este modo, la capacidad de cooperación del MRS está restringida al número de robots que comparten frontera con el objetivo; desaprovechando en gran medida el potencial estratégico brindado por la inclusión de varios robots en la tarea. Nuestro trabajo da solución a esta problemática a través del desplazamiento de los robots hacia sectores en donde tienen la mayor probabilidad de interceptación dentro de su propia zona de operación, situación que puede derivarse en la interceptación directa del objetivo o el bloqueo de espacios con gran número de rutas de acceso desde la posición del objetivo. Lo descrito anteriormente es evidente en las Figuras 8 y 9 , en donde uno de los robots se dirige directamente a interceptar el objetivo, mientras el otro se desplaza y aguarda en una posición estratégica desde donde en caso de fuga, podría brindar una respuesta rápida hacia cualquiera de las posibles trayectorias de salida del intruso.

También, se logró comprobar que la arquitectura de software puede correr en el computador a bordo de los robots, y además, que esta puede ser integrada con su sistema de navegación con el fin de seguir trayectorias evadiendo obstáculos.

\section{CONCLUSIONES}

Se presentó una arquitectura MRS completamente funcional para tareas de interceptación y seguridad en infraestructuras de gran tamaño. Los experimentos muestran que es posible obtener comportamientos cooperativos para interceptar intrusos empleando únicamente las posiciones instantáneas de los robots, la posición del objetivo y el mapa del entorno. La técnica propuesta no emplea trayectorias ni posibles metas para predecir el desplazamiento del intruso, entonces la estimación se realiza para todo el mapa y puede ser aprovechada de mejor forma por el MRS. La distribución dinámica de áreas, mediante el método de Fast Marching, es escalable y eficiente debido a que no incrementa el costo computación con la adición de robots dentro de la distribución. En este sentido, es necesario realizar una adecuada generación del mapa de costos con el fin de mejorar el rendimiento del FMM Core.

Futuros trabajos se orientarán a implementar la red de sensores para obtener medidas reales de la posición de intrusos en el escenario y de esta forma evaluar el comportamiento del MRS cuando el objetivo cambia continuamente de posición. También, se realizarán experimentos y simulaciones exhaustivas para obtener información estadística relevante sobre la eficiencia de la metodología planteada.

\section{Agradecimientos}

Este trabajo fue parcialmente apoyado por el Grupo de Robótica y Cibernética de la Universidad Politécnica de Madrid (España), siendo financiado por los proyectos: PRIC (Protección robotizada de Infraestructuras Criticas; DPI201456985-R), patrocinado por el Ministerio español de Economía y Competitividad y RoboCity2030III-CM (Robótica aplicada a la Mejora de la Calidad de Vida de los Ciudadanos fase III;. S2013 / MIT-2748), financiado por Programas de Actividades de I + D en la Comunidad de Madrid y cofinanciado por Fondos Estructurales de la UE.

\section{Referencias}

[1] Wang, C. Zhang, T. Wang, K. Lv, S. Ma, H. (2013). A new approach of multi-robot cooperative pursuit. Chinese Control Conference. pp. 7252-7256.

[2] Gong, J. Qi, J. Xiong, G. Chen, H. Huang, W. (2007). A GA based combinatorial auction algorithm for multi-robot cooperative hunting. En: Proceedings of the International Conference on Computational Intelligence and Security. pp. $137-141$

[3] Ni, J. Yang, S. X. (2011). Bio-inspired neural network for real-time cooperative hunting by multirobots in unknown environments. IEEE Trans. Neural Netw. 22(12), pp. 2062-2077.

[4] Cai, Z. Sun, L. Gao, H. Zhou, P.(2008). Multirobot cooperative pursuit based on combinatorial auction mechanism under dynamic environment. En: Proceedings of the 2nd Int. Symposium on Systems and Control in Aerospace and Astronautics. pp. 1-6.

[5] Sethian, J. A. (1999). Fast marching methods. SIAM Review, 41(2). pp. 199-235.

[6] Garzón, D. A. Garzón, M. y Barrientos, A. (2016). Pedestrian Motion Prediction: A Graph Based Approach. En: "RoboCity16 Open Conference on Future Trends in Robotics", May 26 27th 2016, Madrid, Spain. pp. 309-316.

[7] Farinelli, A. Iocchi, L. and Nardi, D. (2004). Multirobot systems: a classification focused on coordination. En IEEE Transactions on Systems, Man, and Cybernetics, Part B (Cybernetics). 34(5). pp. 2015-2028.

[8] Huang, H. Zhou, Z. Zhang, W. Ding, J. Stipanovic, D. M. Tomlin, C. J. (2012). Safereachable area cooperative pursuit. IEEE Transactions on Robotics. 